\title{
SYNTHESIS OF (E)-4-METHYL-2-((PHENETHYLIMINO (PHENYL)METHYL)PHENOL AND ITS TRANSITION METAL COMPLEXES, CHARACTERIZATION AND ELECTRICAL CONDUCTIVITY STUDY OF COMPLEXES
}

\author{
A. B. Sahare ${ }^{1}$ and R. B. Mohod $^{2}$ \\ ${ }^{1}$ Department of Chemistry, S.S.E.S. Amravati’s Science College, Pauni Dist. \\ Bhandara-441910, (Maharashtra) India \\ ${ }^{2}$ Department of Chemistry, Shri Shivaji College of Arts, Commerce \& Science, \\ Akola-444001, (Maharashtra) India \\ *E-mail: atulsahare28@gmail.com
}

\begin{abstract}
A novel Schiff base (E)-4-methyl-2-((phenethylimino(phenyl)methyl)phenol synthesized by the condensation of 2hydroxy-5-methylbenzophenone with 2-phenylethylamine. A series of metal complexes of $\mathrm{Mn}(\mathrm{II}), \mathrm{Co}(\mathrm{II}), \mathrm{Ni}(\mathrm{II})$, $\mathrm{Cu}(\mathrm{II}), \mathrm{Zn}(\mathrm{II})$ and $\mathrm{Cd}(\mathrm{II})$ have been prepared with the newly synthesized Schiff base ligand. The synthesized ligand was characterized by elemental analysis, FT-IR and ${ }^{1} \mathrm{H}$ NMR spectra. The complexes have been characterized by different physicochemical techniques. The d.c. electrical conductivity of the synthesized complexes has been measured in compressed pallet form over a wide range of temperatures. The complexes were found to be semiconducting.

Keywords: Schiff Base, FTIR, Diffuse Reflectance, Electrical Conductivity, Transition Metal Complexes, Metal Chelates.
\end{abstract}

(C) RASĀYAN. All rights reserved

\section{INTRODUCTION}

The Schiff bases have a very important role in coordination chemistry. Schiff base metal complexes have attracted great interest in the research field due to novel structural features, interesting thermal, magnetic and spectral properties, biological, industrial and catalytic importance. ${ }^{1-8}$ Many complexes of Schiff base were found to be a very important precursor of semiconducting materials. ${ }^{9,10}$ In the recent past it was reported that the metal complexes of N, O-chelating Schiff base ligands have unusual thermal and electrical stabilities. ${ }^{11,12}$ In our previous publication synthesis and characterization of (E)-4-methyl-2((phenethylimino)(phenyl)methyl) phenol and its $\mathrm{Mn}(\mathrm{II}), \mathrm{Co}(\mathrm{II}), \mathrm{Ni}(\mathrm{II}), \mathrm{Cu}(\mathrm{II}), \mathrm{Zn}(\mathrm{II})$ and $\mathrm{Cd}(\mathrm{II})$ complexes have been reported. ${ }^{13}$ The spectral analysis and d.c. electrical conductivity study of these metal complexes has been reported in the present paper.

\section{EXPERIMENTAL}

\section{Material and Methods}

The solvents and chemicals used in the synthesis were of AR grade and if required, further purified by standard methods. ${ }^{14,15}$

\section{General Procedure}

(a) Preparation of Schiff Base (HMBPE)

Schiff base (E)-4-methyl-2-((phenethylimino)(phenyl)methyl)phenol was prepared by condensation reaction between 2-phenylethylamine and 2-hydroxy-5-methylbenzophonone in ethanol as a solvent. ${ }^{16,17}$ 
RASĀYAN J. Chem.

Vol. 13 | No. 1 |647 - 653| January - March | 2020

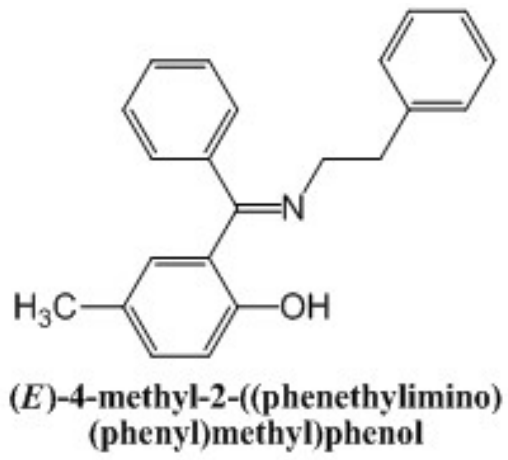

Fig.-1: Proposed Structure of Ligand (HMBPE)

\section{(b) Preparation of Transition Metal Complexes of HMBPE}

A solution of metal(II) acetate was mixed with a solution of ligand HMBPE in DMF in 1:2 (M:L) ratio. ${ }^{18}$ The reaction mixtures were refluxed for 5-6 hours on a sand bath using a water condenser. The colored products obtained were filtered and washed several times with petroleum ether. ${ }^{19}$ Finally, the products were dried by using desiccators over anhydrous $\mathrm{AlCl}_{3}$, then in an electric oven at $60-70^{\circ} \mathrm{C} .^{20}$

\section{Detection Method}

${ }^{1} \mathrm{H}$ NMR spectrum of $\mathrm{HMBPE}$ was recorded in $\mathrm{CDCl}_{3}$ at SAIF, CDRI, Lucknow. FT-IR spectra of all synthesized compounds were recorded in the IR Affinity-1 Shimadzu instrument using $\mathrm{KBr}$ as a reference in the range $400-4000 \mathrm{~cm}^{-1}$. Diffuse reflectance spectra (DRS) were recorded at STIC, Cochin University, Kerala on Varian Cary-5E UV-visible spectrophotometer.

\section{Analytical Discussion}

(a) Elemental Analysis of HMBPE: The elemental analysis suggested $\mathrm{C}_{22} \mathrm{H}_{21} \mathrm{NO}$ empirical formula for the ligand.

(b) The FT-IR spectrum of HMBPE: FT-IR spectrum of HMBPE shows the peaks at $3664 \mathrm{~cm}^{-1}$ (due to phenolic $\mathrm{O}-\mathrm{H}$ stretching), $1608 \mathrm{~cm}^{-1}$ ( $\mathrm{C}=\mathrm{N}$ stretching), $1325 \mathrm{~cm}^{-1}$ (C-O phenolic stretching).

(c) ${ }^{1} \mathrm{H}$ NMR Spectra of $\mathrm{HMBPE}\left(300 \mathrm{MHz}, \mathrm{CdCl}_{3}, \delta\right.$ in $\left.\mathbf{~ p p m}\right)$ : ${ }^{1} \mathrm{H}$ NMR spectrum of ligand shows following peaks ${ }^{21}$ :

$\delta 7.220-7.441(5 \mathrm{H}, \mathrm{m}, \mathrm{Ar}-\mathrm{H}) ; \delta 7.212(1 \mathrm{H}, \mathrm{s}, \mathrm{Ar}-\mathrm{H}) ; \delta 6.919-7.191(5 \mathrm{H}, \mathrm{m}, \mathrm{Ar}-\mathrm{H}) ; \delta 6.876-6.904$ $(1 \mathrm{H}, \mathrm{d}, \mathrm{Ar}-\mathrm{H}) ; \delta 6.484-6.490(1 \mathrm{H}, \mathrm{d}, \mathrm{Ar}-\mathrm{H}) ; \delta 5.542(1 \mathrm{H}, \mathrm{s}$, (broad) -OH); $\delta 3.547-3.595$ (2H, t, $\left.\mathrm{CH}_{2}-\right) ; \delta 2.891-2.990\left(2 \mathrm{H}, \mathrm{t},-\mathrm{CH}_{2}-\right) ; \delta 2.080\left(3 \mathrm{H}, \mathrm{s}, \mathrm{Ar}^{-} \mathrm{CH}_{3}\right) .{ }^{22-25}$

\section{RESULTS AND DISCUSSION}

The compounds synthesized and characterized by FT-IR, elemental analysis, ${ }^{1} \mathrm{H}$ NMR and diffuse reflectance spectra. The physicochemical and spectral data reveals the formation of the complex through donor site azomethine $\mathrm{N}$-atom and phenolic $\mathrm{O}$-atom. The d.c. electrical conductivity study of synthesized complexes has also been carried out.

\section{(1) Elemental Analysis of Compounds:}

The physical and analytical data of compounds are shown in Table-1. Elemental analysis data confirms the stoichiometric composition of the compounds. The data suggest a 1:2 (M:L) ratio in all the complexes. It also indicates the presence of coordinated and lattice water molecules in the complexes. ${ }^{26}$ All the compounds were found to be nonelectrolytes. ${ }^{27}$

\section{(2) FT-IR Spectra}

FT-IR spectral data of HMBPE and its metal complexes are given in Table-2. The spectrum of free HMBPE shows broad band at $3665 \mathrm{~cm}^{-1}$ assigned to phenolic $\mathrm{O}-\mathrm{H}$ stretching. This band was not observed in the spectra of its metal complexes. Another strong absorption band observed at $1628 \mathrm{~cm}^{-1}$ due 
RASĀYAN J. Chem.

Vol. 13 | No. 1 |647 - 653| January - March | 2020

to $\mathrm{C}=\mathrm{N}$ stretching which is shifted to lower frequencies in the spectra of complexes by $26-46 \mathrm{~cm}^{-1}$ suggesting coordination through azomethine nitrogen. ${ }^{29}$

Table-1: Physical and Elemental Analysis Data of Compounds ${ }^{28}$

\begin{tabular}{|c|c|c|c|c|c|c|c|c|}
\hline \multirow{2}{*}{ S. No. } & \multirow{2}{*}{ Complex } & \multirow{2}{*}{ Colour } & \multirow{2}{*}{ Solubility } & \multirow{2}{*}{$\begin{array}{l}\text { Mol. } \\
\text { Wt. }\end{array}$} & \multicolumn{4}{|c|}{$\begin{array}{l}\text { Elemental Analysis \% Found } \\
\text { (Calcd.) }\end{array}$} \\
\hline & & & & & $\mathrm{M}$ & $\mathrm{C}$ & $\mathrm{H}$ & $\mathrm{N}$ \\
\hline 1. & HMBPE & $\begin{array}{l}\text { Pale } \\
\text { Yellow }\end{array}$ & DMF & 315.4 & -- & $\begin{array}{c}83.80 \\
(83.78)\end{array}$ & $\begin{array}{c}6.66 \\
(6.71)\end{array}$ & $\begin{array}{c}4.76 \\
(4.44)\end{array}$ \\
\hline 2. & {$\left[\mathrm{Mn}(\mathrm{HMBPE})_{2}\left(\mathrm{H}_{2} \mathrm{O}\right)_{2}\right]$} & $\begin{array}{c}\text { Light } \\
\text { Brown }\end{array}$ & DMSO & 719.7 & $\begin{array}{c}7.72 \\
(7.63)\end{array}$ & $\begin{array}{c}73.33 \\
(73.42)\end{array}$ & $\begin{array}{c}6.24 \\
(6.16) \\
\end{array}$ & $\begin{array}{c}3.81 \\
(3.89) \\
\end{array}$ \\
\hline 3. & {$\left[\mathrm{Co}(\mathrm{HMBPE})_{2}\left(\mathrm{H}_{2} \mathrm{O}\right)_{2}\right] \cdot \mathrm{H}_{2} \mathrm{O}$} & $\begin{array}{l}\text { Light } \\
\text { Pink }\end{array}$ & DMSO & 741.7 & $\begin{array}{c}7.89 \\
(7.94)\end{array}$ & $\begin{array}{c}71.29 \\
(71.24)\end{array}$ & $\begin{array}{c}6.21 \\
(6.25)\end{array}$ & $\begin{array}{c}3.72 \\
(3.78)\end{array}$ \\
\hline 4. & {$\left[\mathrm{Ni}(\mathrm{HMBPE})_{2}\left(\mathrm{H}_{2} \mathrm{O}\right)_{2}\right] \cdot 2 \mathrm{H}_{2} \mathrm{O}$} & $\begin{array}{l}\text { Light } \\
\text { Green }\end{array}$ & DMSO & 759.5 & $\begin{array}{c}7.76 \\
(7.73) \\
\end{array}$ & $\begin{array}{c}69.68 \\
(69.58) \\
\end{array}$ & $\begin{array}{c}6.43 \\
(6.37) \\
\end{array}$ & $\begin{array}{c}7.68 \\
(7.73) \\
\end{array}$ \\
\hline 5. & {$\left[\mathrm{Cu}(\mathrm{HMBPE})_{2}\right] \cdot \mathrm{H}_{2} \mathrm{O}$} & $\begin{array}{l}\text { Olive } \\
\text { Green }\end{array}$ & DMSO & 710.3 & $\begin{array}{c}8.87 \\
(8.95) \\
\end{array}$ & $\begin{array}{c}74.46 \\
(74.39)\end{array}$ & $\begin{array}{c}5.88 \\
(5.96) \\
\end{array}$ & $\begin{array}{c}3.98 \\
(3.94) \\
\end{array}$ \\
\hline 6. & {$\left[\mathrm{Zn}(\mathrm{HMBPE})_{2}\left(\mathrm{H}_{2} \mathrm{O}\right)_{2}\right] \cdot \mathrm{H}_{2} \mathrm{O}$} & $\begin{array}{l}\text { Light } \\
\text { Gray }\end{array}$ & DMSO & 748.2 & $\begin{array}{c}8.79 \\
(8.74)\end{array}$ & $\begin{array}{c}70.57 \\
(70.63)\end{array}$ & $\begin{array}{c}6.26 \\
(6.20)\end{array}$ & $\begin{array}{l}3.65 \\
(3.74)\end{array}$ \\
\hline 7. & {$\left[\mathrm{Cd}(\mathrm{HMBPE})_{2}\right]$} & $\begin{array}{l}\text { Light } \\
\text { Yellow }\end{array}$ & DMSO & 741.2 & $\begin{array}{c}15.11 \\
(15.17)\end{array}$ & $\begin{array}{c}71.27 \\
(71.30)\end{array}$ & $\begin{array}{c}5.38 \\
(5.44)\end{array}$ & $\begin{array}{c}3.69 \\
(3.78)\end{array}$ \\
\hline
\end{tabular}

The medium intensity band observed at $1325 \mathrm{~cm}^{-1}$ due to phenolic C-O stretching is shifted to lower frequencies in the complexes indicates coordination through deprotonated phenolic oxygen atom. ${ }^{30-32}$ The appearance of new bands in complexes in the range of $529-584 \mathrm{~cm}^{-1}$ and $468-496 \mathrm{~cm}^{-1}$ has been assigned M-O and M-N stretching. ${ }^{33,34}$ The bands in the range of $3367-3445 \mathrm{~cm}^{-1}$ in complexes are due to $v\left(\mathrm{H}_{2} \mathrm{O}\right)$ suggesting hydrated complexes. ${ }^{35}$ The medium intensity sharp bands the range $1507-1531 \mathrm{~cm}^{-1}$ and $806-$ $865 \mathrm{~cm}^{-1}$ suggesting coordinated water molecules in some complexes. ${ }^{36,37}$

Table-2: FT-IR Spectral Data of Compounds $\left(\mathrm{cm}^{-1}\right)$

\begin{tabular}{c|l|c|c|c|c|c|c}
\hline S. No. & \multicolumn{1}{|c|}{ Compound } & $v(\mathrm{O}-\mathrm{H})$ & $v(\mathrm{C}=\mathrm{N})$ & $v(\mathrm{C}-\mathrm{O})$ & $v(\mathrm{M}-\mathrm{O})$ & $v(\mathrm{M}-\mathrm{N})$ & $v\left(\mathrm{H}_{2} \mathrm{O}\right)$ \\
\hline 1. & HMBPE & 3664 & 1628 & 1325 & -- & -- & -- \\
\hline 2. & {$\left[\mathrm{Mn}(\mathrm{HMBPE})_{2}\left(\mathrm{H}_{2} \mathrm{O}\right)_{2}\right]$} & -- & 1602 & 1296 & 532 & 479 & $\begin{array}{c}3418, \\
1531, \\
832\end{array}$ \\
\hline 3. & {$\left[\mathrm{Co}(\mathrm{HMBPE})_{2}\left(\mathrm{H}_{2} \mathrm{O}\right)_{2}\right] \cdot \mathrm{H}_{2} \mathrm{O}$} & -- & 1582 & 1305 & 569 & 496 & $\begin{array}{c}3395, \\
1513, \\
865\end{array}$ \\
\hline 4. & {$\left[\mathrm{Ni}(\mathrm{HMBPE})_{2}\left(\mathrm{H}_{2} \mathrm{O}\right)_{2}\right] \cdot 2 \mathrm{H}_{2} \mathrm{O}$} & -- & 1597 & 1285 & 548 & 485 & $\begin{array}{c}3367, \\
1507, \\
806\end{array}$ \\
\hline 5. & {$\left[\mathrm{Cu}(\mathrm{HMBPE})_{2}\right] \cdot \mathrm{H}_{2} \mathrm{O}$} & -- & 1593 & 1272 & 556 & 468 & 3435 \\
\hline 6. & {$\left[\mathrm{Zn}(\mathrm{HMBPE})_{2}\left(\mathrm{H}_{2} \mathrm{O}\right)_{2}\right] \cdot \mathrm{H}_{2} \mathrm{O}$} & -- & 1589 & 1319 & 529 & 481 & $\begin{array}{c}3445, \\
1530, \\
845\end{array}$ \\
\hline 7. & {$\left[\mathrm{Cd}(\mathrm{HMBPE})_{2}\right]$} & -- & 1586 & 1316 & 584 & 472 & -- \\
\hline
\end{tabular}

(3) Diffuse Reflectance Spectra and d.c. Electrical Conductivity

The diffuse reflectance spectrum of HMBPE complexes of $\mathrm{Mn}$ (II), Co(II) and Ni(II) shows three bands. ${ }^{38}$ These three bands are consistent with octahedral geometry. ${ }^{39-43}$ The absorption bands of $\mathrm{Cu}(\mathrm{II})$ complex corresponds to square planar geometry. ${ }^{44,45} \mathrm{The} \mathrm{Zn}$ (II) and Cd(II) complexes of HMBPE show no d-d transitions. Thus, their geometry cannot be predicted based on diffuse reflectance spectra. ${ }^{46}$ Hence, based on the physicochemical data $\mathrm{Zn}$ (II) complex assigned octahedral and $\mathrm{Cd}$ (II) complex assigned tetrahedral geometry. ${ }^{47}$ The electrical conductivity and diffuse reflectance data are given in Table-3. 
RASĀYAN J. Chem.

Vol. 13 | No. 1 |647 - 653| January - March | 2020

The d.c. electrical conductivity measurements of the complexes were carried out in compressed pellet form at temperature range $303-393 \mathrm{~K}$. The conducting behavior of complexes as a function of temperature was studied using two probe technique. ${ }^{48}$

Table-3: Electrical Conductivity at $373 \mathrm{~K}$ and Diffuse Reflectance Data of the Complexes of HMBPE

\begin{tabular}{|c|c|c|c|c|c|}
\hline \multirow[b]{2}{*}{ S. No. } & \multirow[b]{2}{*}{ Metal Complexes } & \multicolumn{2}{|c|}{ d.c. Electrical Conductivity } & \multicolumn{2}{|c|}{ Diffuse Reflectance } \\
\hline & & $\begin{array}{c}\Sigma \\
\left(\Omega^{-1} \mathrm{~cm}^{-1}\right)\end{array}$ & $\begin{array}{c}\mathrm{Ea} \\
(\mathrm{eV})\end{array}$ & $\begin{array}{l}\text { Abs. Band } \\
\left(\mathrm{cm}^{-1}\right)\end{array}$ & Assignments \\
\hline 1. & {$\left[\mathrm{Mn}(\mathrm{HMBPE})_{2}\left(\mathrm{H}_{2} \mathrm{O}\right)_{2}\right]$} & $3.15 \times 10^{-9}$ & 0.139 & $\begin{array}{l}17578 \\
23035 \\
25053 \\
\end{array}$ & $\begin{array}{c}{ }^{6} \mathrm{~A}_{1 \mathrm{~g}} \rightarrow{ }^{4} \mathrm{~T}_{1 \mathrm{~g}}\left({ }^{4} \mathrm{G}\right) \\
{ }^{6} \mathrm{~A}_{1 \mathrm{~g}} \rightarrow{ }^{4} \mathrm{~T}_{2 \mathrm{~g}}\left({ }^{4} \mathrm{G}\right) \\
{ }^{6} \mathrm{~A}_{1 \mathrm{~g}} \rightarrow{ }^{4} \mathrm{E}_{\mathrm{g}}\end{array}$ \\
\hline 2. & {$\left[\mathrm{Co}(\mathrm{HMBPE})_{2}\left(\mathrm{H}_{2} \mathrm{O}\right)_{2}\right] \cdot \mathrm{H}_{2} \mathrm{O}$} & $9.23 \times 10^{-10}$ & 0.248 & $\begin{array}{l}15936 \\
19120 \\
28248 \\
\end{array}$ & $\begin{array}{c}{ }^{4} \mathrm{~T}_{1 \mathrm{~g}}(\mathrm{~F}) \rightarrow{ }^{4} \mathrm{~T}_{2 \mathrm{~g}}(\mathrm{~F}) \\
{ }^{4} \mathrm{~T}_{1 \mathrm{~g}}(\mathrm{~F}) \rightarrow{ }^{4} \mathrm{~A}_{2 \mathrm{~g}}(\mathrm{~F}) \\
{ }^{4} \mathrm{~T}_{1 \mathrm{~g}}(\mathrm{~F}) \rightarrow{ }^{4} \mathrm{~T}_{1}(\mathrm{P})\end{array}$ \\
\hline 3. & {$\left[\mathrm{Ni}(\mathrm{HMBPE})_{2}\left(\mathrm{H}_{2} \mathrm{O}\right)_{2}\right] \cdot 2 \mathrm{H}_{2} \mathrm{O}$} & $2.83 \times 10^{-8}$ & 0.106 & $\begin{array}{l}10131 \\
15337 \\
28169 \\
\end{array}$ & $\begin{array}{c}{ }^{3} \mathrm{~A}_{2 \mathrm{~g}}(\mathrm{~F}) \rightarrow{ }^{3} \mathrm{~T}_{2 \mathrm{~g}}(\mathrm{~F}) \\
{ }^{3} \mathrm{~A}_{2 \mathrm{~g}}(\mathrm{~F}) \rightarrow{ }^{3} \mathrm{~T}_{1 \mathrm{~g}}(\mathrm{~F}) \\
{ }^{3} \mathrm{~A}_{2 \mathrm{~g}}(\mathrm{~F}) \rightarrow{ }^{3} \mathrm{~T}_{1 \mathrm{~g}}(\mathrm{P})\end{array}$ \\
\hline 4. & {$\left[\mathrm{Cu}(\mathrm{HMBPE})_{2}\right] \cdot \mathrm{H}_{2} \mathrm{O}$} & $2.26 \times 10^{-10}$ & 0.311 & $\begin{array}{l}14025 \\
19120 \\
37594 \\
\end{array}$ & $\begin{array}{c}{ }^{2} \mathrm{~B}_{1 \mathrm{~g}} \rightarrow{ }^{2} \mathrm{~A}_{1 \mathrm{~g}} \\
{ }^{2} \mathrm{~B}_{1 \mathrm{~g}} \rightarrow{ }^{2} \mathrm{E}_{\mathrm{g}} \\
\text { C. } \mathrm{T} .\end{array}$ \\
\hline 5. & {$\left[\mathrm{Zn}(\mathrm{HMBPE})_{2}\left(\mathrm{H}_{2} \mathrm{O}\right)_{2}\right] \cdot \mathrm{H}_{2} \mathrm{O}$} & $3.17 \times 10^{-8}$ & 0.195 & $\begin{array}{l}26315 \\
36231 \\
\end{array}$ & $\begin{array}{c}\text { C.T. } \\
\pi \rightarrow \pi^{*}\end{array}$ \\
\hline 6. & {$\left[\mathrm{Cd}(\mathrm{HMBPE})_{2}\right]$} & $1.93 \times 10^{-7}$ & 0.279 & $\begin{array}{l}28248 \\
30211 \\
38910\end{array}$ & $\begin{array}{c}\text { C.T. } \\
\mathrm{n} \rightarrow \pi^{*} \\
\pi \rightarrow \pi^{*}\end{array}$ \\
\hline
\end{tabular}

Solid-state d.c. electrical conductivity of HMBPE metal complexes was found in range of $9.23 \times 10^{-10}$ to $1.93 \times 10^{-7} \Omega^{-1} \mathrm{~cm}^{-1}$ at $373 \mathrm{~K}$ and decreases in order $\mathrm{Cd}(\mathrm{II})>\mathrm{Zn}(\mathrm{II})>\mathrm{Ni}(\mathrm{II})>\mathrm{Mn}(\mathrm{II})>\mathrm{Co}(\mathrm{II})>$ $\mathrm{Cu}(\mathrm{II}) .^{49,50}$ It has been found that the electrical conductivity of HMBPE complexes of Mn(II), Co(II), $\mathrm{Cu}(\mathrm{II})$ and $\mathrm{Zn}$ (II) increases with increasing temperature and decreases with cooling in temperature range under study, indicating the semiconducting behaviour. ${ }^{51}$ The d.c. electrical conductivity $(\sigma)$ has been found to vary according to Arrhenius equation: ${ }^{52-54}$

$$
\sigma=\sigma_{0} \exp \left(-\mathrm{E}_{\mathrm{a}} / \mathrm{kT}\right)
$$

Where, $\quad \sigma_{0}$ - Conductivity constant,

$$
\mathrm{k} \text { - Boltzmann's constant, }
$$

$E_{a}$ - Activation energy,

$\mathrm{T}$ - Absolute temperature.

The plot of $\log \sigma$ vs. 1000/T of complexes was found to be linear over the temperature range 303-393 K (Fig.-2). The electrical conductance of $\mathrm{Ni}(\mathrm{II})$ complex was found to decrease with an increase in temperature up to temperature $343 \mathrm{~K}$ and above this, it increases with temperature. This indicates the initial behavior was like a typical metallic conductor while the latter behavior was the characteristics of semiconductor. ${ }^{55}$ The plot of $\mathrm{Cd}$ (II) complex shows two different regions. In the low-temperature range, a slow increase in electrical conductivity and high-temperature range rapid increase with temperature has been observed. The activation energy $\left(E_{a}\right)$ of HMBPE metal complexes obtained from plots was found in the range $0.106-0.311 \mathrm{eV}$ and decrease in order $\mathrm{Cu}(\mathrm{II})>\mathrm{Cd}(\mathrm{II})>\mathrm{Co}(\mathrm{II})>\mathrm{Zn}(\mathrm{II})>\mathrm{Mn}(\mathrm{II})>\mathrm{Ni}(\mathrm{II})^{56,57}$. Activation energy is the direct measure of the band gap of semiconductors, lowers the activation energy, the lower will be the band gap. ${ }^{58}$

\section{CONCLUSION}

The metal complexes of Schiff base ligand HMBPE have been synthesized and characterized by different analytical and spectroscopic methods. Solid-state d.c. the electrical conductivity of HMBPE metal complexes has been measured at different temperatures in compressed pellet form. ${ }^{59}$ The study shows semiconducting behavior of HMBPE metal complexes in the range of temperature 303-393 K. The activation energy of electrical conduction of the complexes was found in the range $0.106-0.311 \mathrm{eV}$ in the order of $\mathrm{Cu}(\mathrm{II})>\mathrm{Cd}(\mathrm{II})>\mathrm{Co}(\mathrm{II})>\mathrm{Zn}(\mathrm{II})>\mathrm{Mn}(\mathrm{II})>\mathrm{Ni}(\mathrm{II})$. 
RASĀYAN J. Chem.

Vol. 13 | No. 1 |647 - 653| January - March | 2020

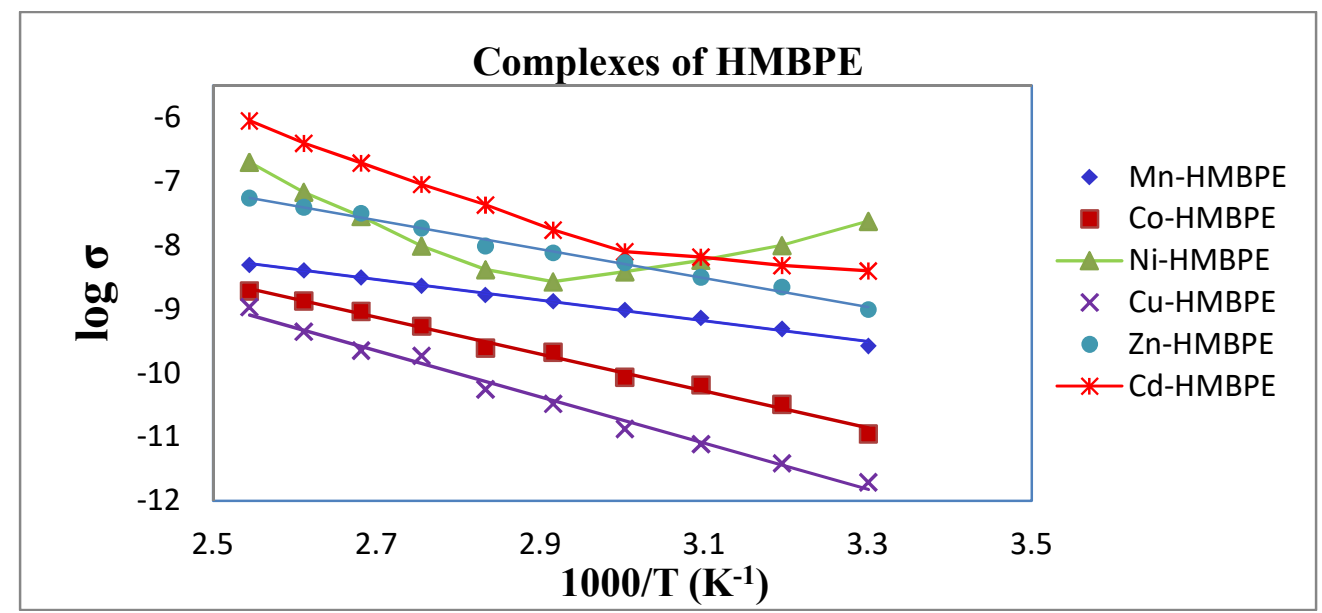

Fig.-2: Variation of Electrical Conductivity of HMBPE Complexes with Temperature

\section{ACKNOWLEDGMENT}

The authors are very thankful to SAIF-STIC, Cochin (Kerala) and SAIF, CDRI, Lucknow for providing spectral data. Also, thankful to Principal, Shri Shivaji College of Arts, Commerce and Science, Akola for providing necessary facilities.

\section{REFERENCES}

1. Z. Dobrokhotova, A. Emelina, A. Sidorov, G. Aleksandrov, M. Kiskin, P. Koroteev, M. Bykov, M. Fazylbekov, A. Bogomyakov, V. Novotortsev and I. Eremenko, Polyhedron, 30(1), 132(2011), DOI:10.1016/j.poly.2010.09.040

2. J. Bavane and R. Mohod, Journal of Chemical and Pharmaceutical Research, 8(3), 913(2016).

3. F. A. Cotton, G. Wilkinson and C. A. Murillo, Advanced Inorganic Chemistry; Wiley-Interscience: New York, 23 (1999).

4. J. Costamagna, J. Vargas and R. Lactorre, Coordination Chemistry Review, 119, 67(1992).

5. L. H. Abdel-Rahman, R. M. El-Khatib, L. A. E. Nassr, A. M. Abu-Dief, F. El-Din Lashin, Spectrochimica Acta Part A: Molecular and Biomolecular Spectroscopy, 111, 266(2013), DOI: 10.1016/j.saa.2013.03.061

6. S. Priyarega, R. Prabhakaran, K. R. Aranganayagam, R. Karvembu and K. Natarajan, Applied Organometallic Chemistry, 21, 788(2007), DOI:10.1002/aoc.1281

7. D. Chen and A. E. Martel, Inorganic Chemistry, 26, 1026(1987), DOI:10.1021/ic00254a013

8. S. Begic, N. Ljubijankic, S. Gojak-Salimovic and E. Osmic, Rasayan Journal of Chemistry, 12(1), 294(2019), DOI:10.31788/RJC.2019.1215112

9. H. Hassib and A. A. Razik, Solid State Communication, 147, 345(2008), DOI: 10.1016/j.ssc.2008.06.034

10. S. Sarkar, V. Aydogdu, F. Dagdelen, B. B. Bhaumik, K. Dey, Material Chemistry and Physics, 88, 357 (2004), DOI:10.1016/j.matchemphys.2004.08.001

11. H. S. Abbo, S. J. J. Titinchi, R. Prasad and S. Chaand, Molecular Catalysis, 225(2), 225(2005), DOI:10.1016/j.molcata.2004.09.007

12. S. Djebbar-Sid and O. Benali-Baitic, Transition Metal Chemistry, 23, 443(1998).

13. A. B. Sahare and R. B. Mohod, International Journal of Current Engineering and Scientific Research, 6(1), 1377(2019).

14. H. Maradiya and V. Patel, Journal of Fibers and Polymers, 3, 314(2002).

15. L. H. Abdel-Rahman, A. M. Abu-Dief, M. O. Aboelez, A.A. Hassan Abdel-Mawgoud, Journal of Photochemistry and Photobiology B, 170, 271(2017), DOI:10.1016/j.jphotobiol.2017.04.003

16. R. K. Mohapatra, P. K. Das, M. K. Pradhan, A. A. Maihub, M. M. El-ajaily, Journal of the Iranian Chemical Society, 15(5), 2193(2018), DOI:10.1007/s13738-018-1411-2

17. N. Ljubijankic, S. Begic, A. Ljubovic-Dedeic, M. Stankovic, I. Salimovic-Bešic, M. Jadranin, B. Bencun and S. Ljubijankic, Rasayan Journal of Chemistry, 11(4), 1511(2018), 
RASĀYAN J. Chem.

Vol. 13 | No. 1 |647 - 653| January - March | 2020

DOI: $10.31788 /$ RJC.2018.1145021

18. Nabil S. Youssef, Eman A. El-Zahany, Ahmed M. A. El-Seidy, Phosphorus, Sulfur and Silicon and the Related Elements, (4):785(2010), DOI:10.1080/10426500902967904

19. Mishra, Monika, Karishma Tiwari, Sachin Shukla, R. Mishra, and Vinod P. Singh, Spectrochimica Acta Part A Molecular and Biomolecular Spectroscopy, 132C, 452(2014), DOI:10.1016/j.saa.2014.05.007

20. Saied M. E. Khalil, Journal of Coordination Chemistry, 56(12), 1013(2003), DOI:10.1080/0095897031000135289

21. Mehmet Sonmez, Synthesis and Reactivity in Inorganic Metal-Organic and Nano-Metal Chemistry, 34(3), 489(2005), DOI:10.1081/SIM-120030436

22. B. Naik and K. R. Desai, Indian Journal of Chemistry, 45B, 267(2006).

23. E. J. Campbell and S. T. Nguyen, Tetrahedron Letters, 42(7), 1221(2001), DOI:10.1016/S00404039(00)02226-7

24. B. Narayana, K. K. Vijaya Raj, B. V. Ashalatha, N. Suchetha Kumari, B. K. Sarojini, European Journal of Medicinal Chemistry, 39(10), 867(2004), DOI: 10.1016/j.ejmech.2004.06.003

25. Won Suck Sun, Yoon Sun Park, Jakyung Yoo, Ki Duk Park, Sung Han Kim, Jung-Han Kim, and Hyun-Ju Park, Journal of Medicinal Chemistry, 46(26), 5619(2003), DOI:10.1021/jm0205346

26. Arish, D., Sivasankaran Nair M., Journal of Molecular Structure, 983(1), 112(2010), DOI:10.1016/j.molstruc.2010.08.040

27. W. J. Geary, Coordination Chemistry Review, Elsevier Publishing Company. 7, 81(1971), DOI:10.1016/S0010-8545(00)80009-0

28. Mahmoud M. Mashaly, Z. H. Abd-Elwahab and A. A. Faheim, Synthesis and Reactivity in Inorganic Metal-Organic and Nano-Metal Chemistry, 34(2), 235(2004), DOI:10.1081/SIM120028300

29. G. S. Sanyal, P. K. Nath and R. Ganguly, Journal of the Indian Chemical Society, 79, 54 (2002).

30. M. R. Maurya, D. C. Antony, S. Gopinathan, C. Gopinathan, Bulletin of Chemical Society Japan., 68, 554 (1995), DOI:10.1246/bcsj.68.554

31. P. K. Panchal and M. N. Patel, Synthesis and Reactivity in Inorganic Metal-Organic and NanoMetal Chemistry, 34(7), 1277(2004), DOI:10.1081/SIM-120039271

32. Debdas Mandal and Bikramaditya Mandal, Rasayan Journal of Chemistry, 12(2), 754(2019), DOI: 10.31788/RJC.2019.1225230

33. B. S. Garg and D. N. Kumar, Spectrochimica Acta, 59A(2), 229(2003), DOI:10.1016/S1386$1425(02) 00142-7$

34. N. S. Bhave and A. S. Aswar, Asian Journal of Chemistry, 4(1), 65 (1992).

35. Shashidhar, K. Shivakumar, P. Vithal Reddy and M. P. Halli, Journal of Coordination Chemistry, 60(3), 243(2007), DOI:10.1080/00958970601112051

36. B. S. Patel and S. R. Patel, Macromolecular Chemistry and Physics, 180, 1159(1979), DOI: $10.1002 / \mathrm{macp} .1979 .021800504$

37. Shariful Islam, A. K. M. Nur Alam Siddiki, Shahida Begum, Md. Abdus Salam, Open Journal of Inorganic Chemistry, 08(02), 55(2018), DOI: 10.4236/ojic.2018.82005

38. M. S. Masoud, A. Kh. Ghonam, R. H. Ahmed, S. A. Aboud El-Enein and A. A. Mahmoud, Journal of Coordination Chemistry, 55(1), 79(2002), DOI:10.1080/00958970211870

39. M. Padmaja, J. Pragathi, B. Anupama and C. Gyana Kumari, E-Journal of Chemistry, 9(4), 2145(2012), DOI: $10.1155 / 2012 / 839789$

40. R. K. Agarwal, L. Singh, D. K. Sharma and R. Singh, Turk Journal of Chemistry, 29, 309(2005).

41. K. Ueno and A. E. Martel, The Journal of Physical Chemistry, 61(3), 257(1957), DOI:10.1021/ j150549a001

42. U. Casellato, D. Fregona, S. Sitran, S. Tamburini, and P. A. Vigato, Inorganica Chimica Acta, 95, 306(1984), DOI:10.1016/S0020-1693(00)84899-9

43. Y. Satyawana, R. Meena, R. V. Singh and N. Fahmi, Rasayan Journal of Chemistry, 12(4), 2328 (2019), DOI:10.31788/RJC.2019.1245459

44. J. C. Bailer, H. J. Emeleus, R. Nyholm and A. F. Trotman-Dickinson, Comprehensive Inorganic 
RASĀYAN J. Chem.

Vol. 13 | No. 1 |647 - 653| January - March | 2020

Chemistry; Pergamon Press: Oxford, UK (1975).

45. A.B.P. Lever, Inorganic Electronic Spectroscopy (2nd Ed.). Elsevier: Amsterdam, (1984).

46. A. Majumder, G. M. Rosair, A. Mallick, N. Chattopadhyay and S. Mitra, Polyhedron, 25(8), 1753(2006), DOI:10.1016/j.poly.2005.11.029

47. Gary A. Foulds, Coordination Chemistry Reviews, 142, 153(1995), DOI:10.1016/00108545(95)96888-L

48. K. Mohanan, N. Subhadrambika, R. Selwin Joseyphus, S. S. Swathy and V. P. Nisha, Journal of Saudi Chemical Society, 20(4), 379(2016), DOI:10.1016/j.jscs.2012.07.007

49. Neelu Chouhan, M. L. Kalra, Sudhish Kumar, Y. N. Singh and Suresh C. Ameta, Molecular Crystals and Liquid Crystals, 469(1), 99(2007), DOI:10.1080/15421400701431778

50. J. D. Joshi, S. D. Patel and G. P. Patel, Journal of Macromolecular Science, Part A, 44(1), 65(2007), DOI:10.1080/10601320601044500

51. A. K. Maldhure and A. S. Aswar, Journal of the Indian Chemical Society, 86(7), 697(2009).

52. M. S. Masoud, S. A. El-Enein, and E. El-Shereafy, Journal of Thermal Analysis, 37, 365(1991), DOI: $10.1007 / \mathrm{BF} 02055938$

53. M. G. Abd El Wahed, K. A. El Manakhly and A. Amer, Journal of Materials Science Letters, 15(11), 919(1996)

54. A. S. Aswar and N. S. Bhave, Asian Journal of Chemistry, 2(4), 363 (1990).

55. A. S. Aswar and N. S. Bhave, Colloid and Polymer Science, 269(6), 547(1991), DOI: 10.1007/BF00659907

56. Gaurav B. Pethe, Vijaya V. Dhande, Prashant R. Mandlik, Anand S. Aswar, Reviews in Inorganic Chemistry, 29(4), 225(2009), DOI:10.1515/REVIC.2009.29.4.225

57. N. Deb, N. N. Dass and P. K. Gogoi, Thermochimica Acta, 198, 395(1992).

58. A. A. Shabana, K. A. El-Manakhly and H. A. Hammad, Canadian Journal of Applied Spectroscopy., 39, 22 (1994).

59. T. A. Reena, E. B. Seena and M. R. Prathapachandra Kurup, Polyhedron, 27, 1825(2008), DOI:10.1016/j.poly.2008.02.020

[RJC-5525/2019] 